\title{
Uncertainty in the delayed neutron fraction in fuel assembly depletion calculations
}

\author{
Alexander Aures ${ }^{1, \text { a }}$, Friederike Bostelmann ${ }^{1}$, Ivan A. Kodeli ${ }^{2}$, Kiril Velkov $^{1}$, and Winfried Zwermann ${ }^{1}$ \\ ${ }^{1}$ Gesellschaft für Anlagen- und Reaktorsicherheit (GRS) gGmbH, Boltzmannstraße 14, 85748 Garching, Germany \\ 2 Jožef Stefan Institute, Jamova cesta 39, 1000 Ljubljana, Slovenia
}

\begin{abstract}
This study presents uncertainty and sensitivity analyses of the delayed neutron fraction of light water reactor and sodium-cooled fast reactor fuel assemblies. For these analyses, the sampling-based XSUSA methodology is used to propagate cross section uncertainties in neutron transport and depletion calculations. Cross section data is varied according to the SCALE 6.1 covariance library. Since this library includes nubar uncertainties only for the total values, it has been supplemented by delayed nu-bar uncertainties from the covariance data of the JENDL-4.0 nuclear data library. The neutron transport and depletion calculations are performed with the TRITON/NEWT sequence of the SCALE 6.1 package. The evolution of the delayed neutron fraction uncertainty over burn-up is analysed without and with the consideration of delayed nu-bar uncertainties. Moreover, the main contributors to the result uncertainty are determined. In all cases, the delayed nu-bar uncertainties increase the delayed neutron fraction uncertainty. Depending on the fuel composition, the delayed nu-bar values of uranium and plutonium in fact give the main contributions to the delayed neutron fraction uncertainty for the LWR fuel assemblies. For the SFR case, the uncertainty of the scattering cross section of $\mathrm{U}-238$ is the main contributor.
\end{abstract}

\section{Introduction}

Delayed neutrons affect the dynamic behaviour of a nuclear reactor. Therefore, an accurate determination of the delayed neutron fraction $\beta$ and its uncertainty is of crucial importance for the purpose of reactor transient and safety analyses.

The importance of calculating kinetic parameters and propagating their uncertainties to reactor core simulations was emphasized in the framework of the Uncertainty Analysis in Modelling (UAM) project [1,2] of the OECD/NEA. This has led to efforts for evaluating delayed neutron fractions and their uncertainties with deterministic and stochastic tools (see, e.g. [3]) for various arrangements, mainly critical assemblies documented in the International Handbook of Evaluated Criticality Safety Benchmark Experiments ("ICSBEP Handbook") [4] and the International Handbook of Evaluated Reactor Physics Benchmark Experiments ("IRPhEP Handbook") [5], for which delayed neutron fractions were determined in the experiments.

Such critical experiments are not necessarily representative for large power reactor cores in operational conditions, where the temperatures are usually significantly higher, and the fuel undergoes changes in its isotopic composition in the course of time due to depletion. Therefore, in the present contribution, the fuel assembly depletion calculations have been performed at representative conditions. These serve for the generation of few-group macroscopic cross sections and kinetic parameters including their uncertainties which eventually can be used in subsequent full-core simulations.

a e-mail: Alexander. Aures@grs.de

\section{Methods, codes, and data}

For the current analyses, the sampling-based XSUSA ("Cross Section Uncertainty and Sensitivity Analysis") methodology [6] is used to propagate the cross section uncertainties in neutron transport and depletion calculations. XSUSA uses probability distributions to sample the cross sections of all relevant reactions of all isotopes in every energy group in order to generate random variations of these cross sections. This means that many nuclear data libraries are generated, where all quantities with available uncertainties, namely inelastic and elastic scattering, $(n, 2 n)$ and capture cross sections, in the case of fissionable nuclides additionally the fission cross section, the average number of neutrons per fission, and the fission neutron spectrum, are being varied. A sample size of 1,000 was chosen to allow the determination of about five main contributors to the uncertainty, see below. The uncertainties, along with correlations among them, are taken from the covariance library in 44-group representation as included in the SCALE 6.1 code package [7]. Uncertainties of the numbers of both the prompt and delayed neutrons released per fission event (prompt and delayed "nu-bar") are not individually included there, but only uncertainties of the total nu-bar. Since the delayed nubar uncertainties may substantially affect the uncertainty in the delayed neutron fraction, these uncertainties have additionally been taken from the covariance data of the JENDL-4.0 [8] nuclear data library.

In Figs. 1-3, the uncertainty profiles for the numbers of the total and delayed neutrons released per fission event are displayed for U-235, U-238, and Pu-239, where, as already mentioned, the total values are those provided with SCALE 6.1, while the delayed values are

(C) The Authors, published by EDP Sciences. This is an Open Access article distributed under the terms of the Creative Commons Attribution License 4.0 (http://creativecommons.org/licenses/by/4.0/). 


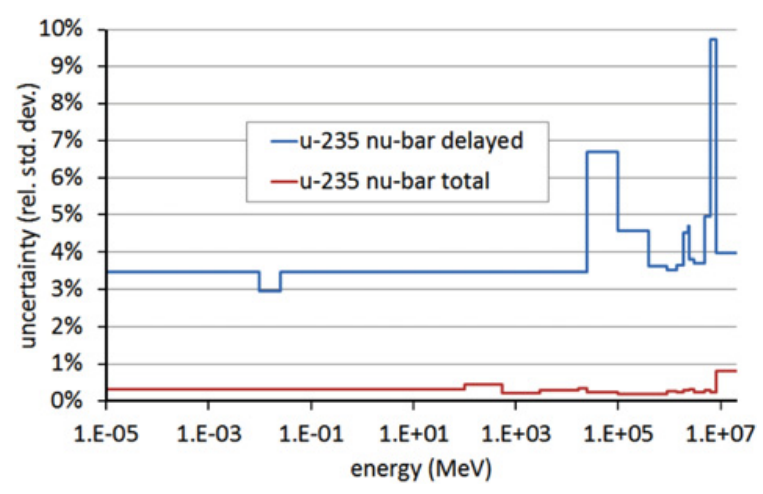

Figure 1. Uncertainty of total and delayed nu-bar of U-235 as a function of neutron energy.

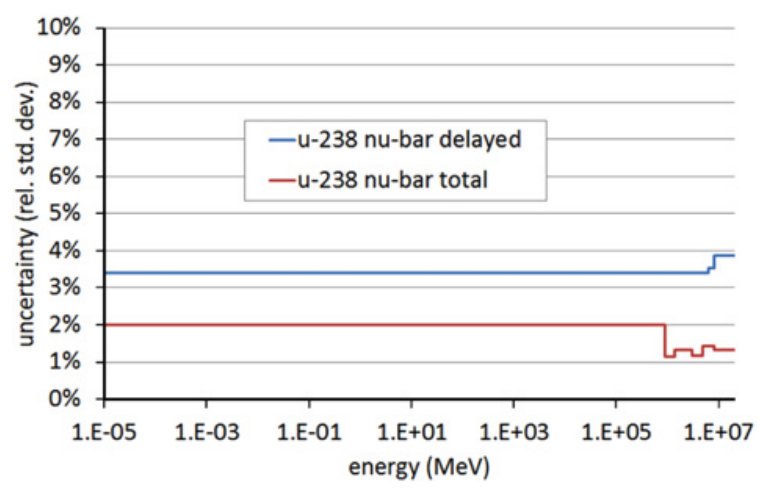

Figure 2. Uncertainty of total and delayed nu-bar of U-238 as a function of neutron energy.

processed from JENDL-4.0. It is seen that in particular for U-235 and $\mathrm{Pu}-239$, the uncertainties of the delayed nu-bar are substantially larger than the corresponding uncertainties for the total nu-bar; this implies that using individual uncertainties for the delayed nu-bar will lead to significantly larger uncertainties for output quantities sensitive to delayed nu-bar as compared to the case where only total nu-bar uncertainties are considered.

The neutron transport and depletion calculations have been performed with the TRITON/NEWT sequence of SCALE 6.1 with a 238-group cross section library based on ENDF/B-VII.0. TRITON/NEWT edits the delayed neutron fractions in the traditional six-group representation; for the subsequent analyses, these values have been summed up to obtain single values for the delayed neutron fractions for every burn-up step.

The evolution of the delayed neutron fraction uncertainty as a function of burn-up has been analysed without and with the consideration of delayed nu-bar uncertainties. Moreover, the main contributors to the result uncertainty are investigated for the beginning of cycle (BOC) and the end of cycle (EOC). For estimating the main contributions to the result uncertainties, one can determine the group sensitivities, where typically the nuclear multi-group data for a certain reaction of a certain nuclide are treated as one group. The group sensitivity analysis is performed by determining the squared multiple correlation coefficient $\mathrm{R}^{2}$ as uncertainty importance indicator to quantify the uncertainty importance of a group of input variables with respect to an output variable [9]. $\mathrm{R}^{2}$ can be interpreted as the relative amount of output

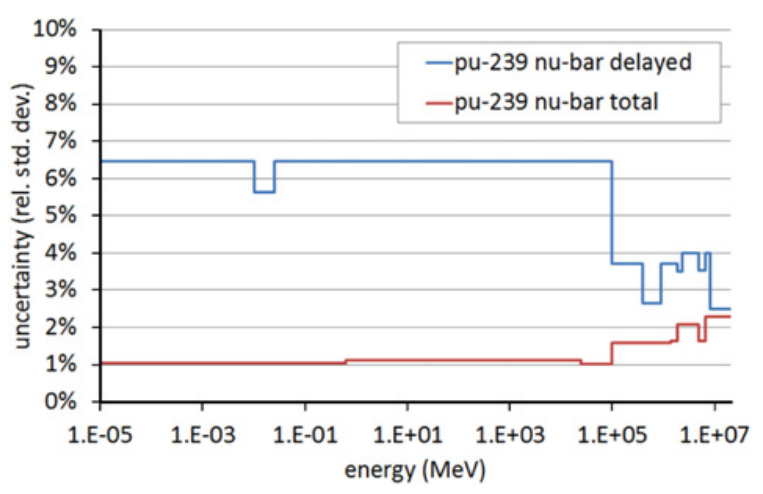

Figure 3. Uncertainty of total and delayed nu-bar of Pu-239 as a function of neutron energy.

variance coming from the uncertainty of the respective parameter group.

\section{The fuel assemblies}

Uncertainty and sensitivity analyses of the delayed neutron fraction have been performed for the fuel assemblies which are representative for light water reactors (LWR) currently in operation, as well as for a fuel assembly of a sodiumcooled fast reactor (SFR). For the former, a pressurized water reactor (PWR) and a boiling water reactor (BWR) fuel assembly have been chosen. The PWR fuel assembly, taken from the specification of the UAM-LWR benchmark phase II [10], is a standard square design with $15 \times 15$ fuel pins with 17 control rod guide tubes, with $\mathrm{UO}_{2}$ fuel with a U-235 enrichment of $4.12 \%$, depleted at a power density of $33.75 \mathrm{MW} / \mathrm{tHM}$ up to a burn-up of 40 GWd/tHM (tHM reads metric ton of initial heavy metal). The BWR fuel assembly, taken from an OECD/NEA benchmark performed in the framework of the Working Party on the Physics of Plutonium Fuels and Innovative Fuel Cycles [11], is a rather heterogeneous square design with 10x10 fuel pins, nine of which are replaced by a central water channel ("atrium"). The fuel assembly consists of $\mathrm{UO}_{2} / \mathrm{Gd}$ and different MOX fuel rods, depleted at a power density of $25.0 \mathrm{MW} / \mathrm{tHM}$ up to a burn-up of $40 \mathrm{GWd} / \mathrm{tHM}$. The SFR fuel assembly is taken from the SFR-FT benchmark [12] specification; it corresponds to one of the fuel assemblies of the large oxide core described there. It is of hexagonal shape with 271 fuel pins, mainly containing plutonium and U-238. The fuel composition at the beginning of the burn-up cycle is chosen such that it is representative of the equilibrium core, including a certain amount of molybdenum as replacement for the fission products. The fuel is depleted at $50.4 \mathrm{MW} / \mathrm{tHM}$ for 410 days, corresponding to a burn-up of $20.66 \mathrm{GWd} / \mathrm{tHM}$.

\section{Results}

This section summarizes the results for the calculated values of the delayed neutron fractions as a function of operation time, along with the corresponding uncertainty and sensitivity analyses.

\subsection{Nominal values}

Nominal values of the delayed neutron fraction as a function of operation time are given in Fig. 4. All 


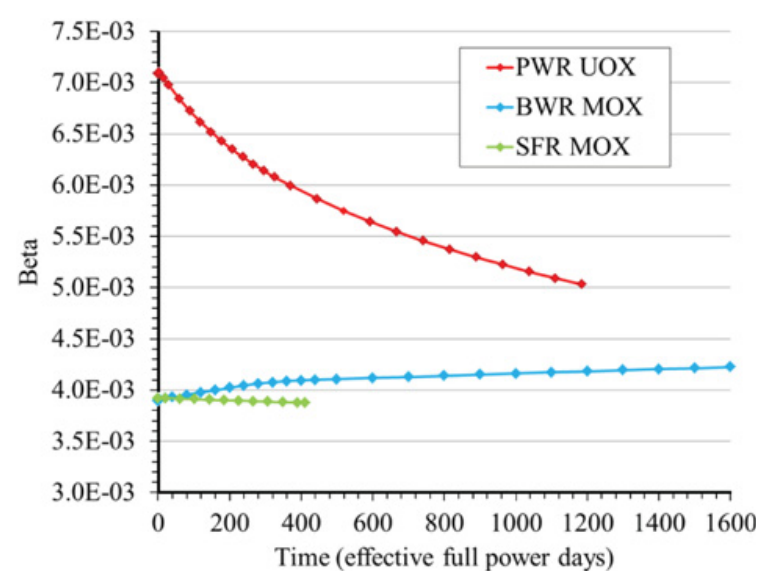

Figure 4. $\beta$ as a function of operation time for the studied fuel assemblies.

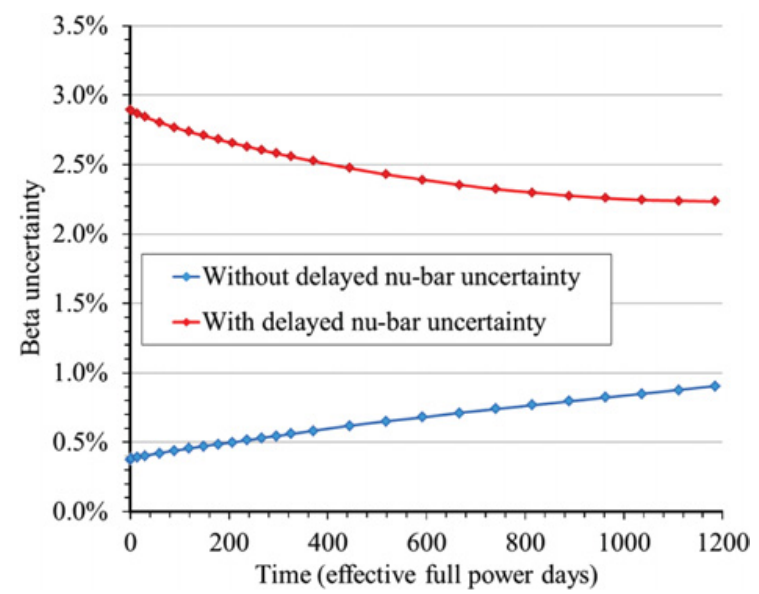

Figure 5. Relative $1 \sigma$ uncertainties in $\beta$ as a function of operation time for the PWR $\mathrm{UO}_{2}$ fuel assembly.

the values qualitatively show the expected behaviour. The BWR and SFR fuel assemblies are dominated by plutonium over their lifetime; therefore, the values of the delayed neutron fractions start at approximately 0.004 and do not change much during burn-up. The PWR fuel assembly contains low-enriched uranium at the beginning, and plutonium is built up during burn-up; therefore, the value starts at approximately 0.007 and continuously decreases during burn-up.

\subsection{Uncertainty analysis}

The uncertainties in the delayed neutron fractions as functions of the operation time for the three fuel assemblies are displayed in Figs. 5-7.

It can be seen that for both LWR fuel assemblies, the inclusion of the uncertainties in the average number of delayed neutrons per fission increases the uncertainties in the delayed neutron fractions by more than a factor of two; for the PWR fuel assembly, the increase during about the first half of its lifetime is even substantially higher. Later on, the uncertainties for the PWR and BWR assemblies become similar. The decrease of the uncertainties for the PWR may be attributed to the (nonconservative) assumption of no correlation among the delayed nu-bar uncertainties of different isotopes. For the BWR assembly, the uncertainties do not change much during its lifetime. This behaviour is due to the fact that

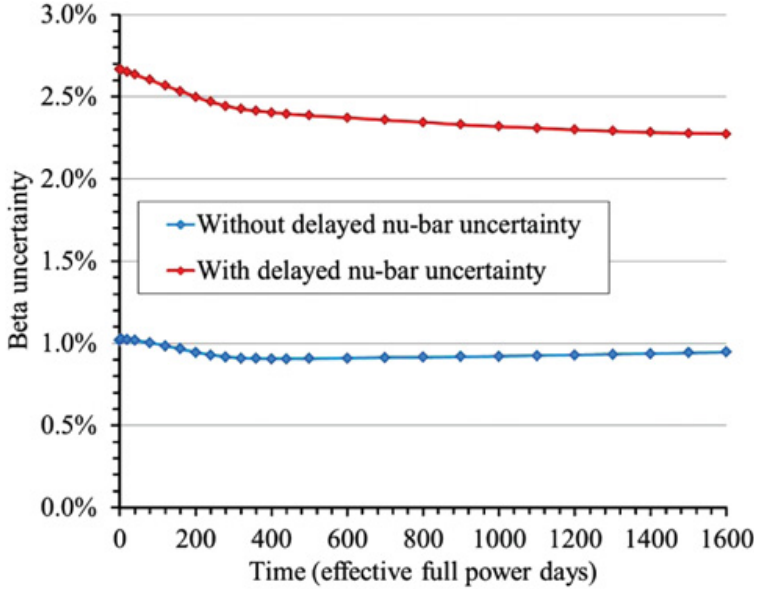

Figure 6. Relative $1 \sigma$ uncertainties in $\beta$ as a function of operation time for the BWR MOX fuel assembly.

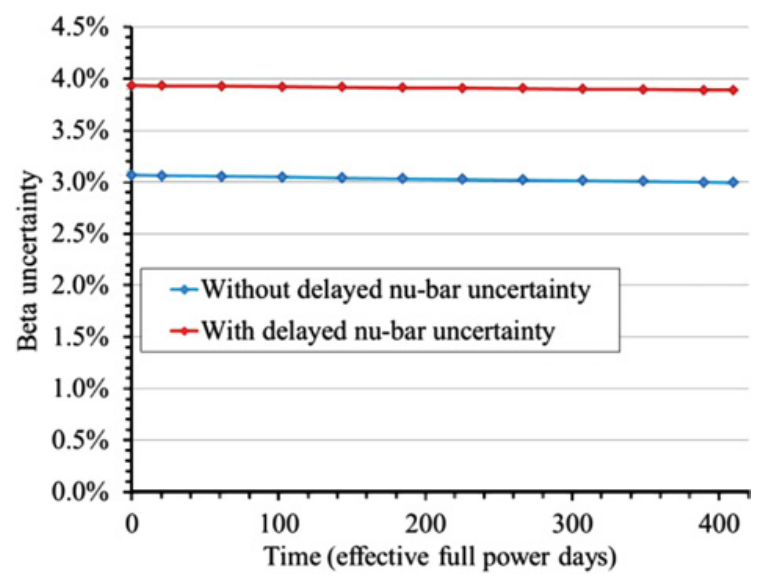

Figure 7. Relative $1 \sigma$ uncertainties in $\beta$ as a function of operation time for the SFR MOX fuel assembly.

initially the fresh BWR assembly has mainly plutonium as fissile material, whereas the PWR assembly initially contains U-235, which is depleted during burn-up while plutonium is being built up.

For the SFR fuel assembly, the situation is quite different. Here again, the burn-up dependence of the uncertainties is weak due to the fact that the isotopic inventory only slightly changes with burn-up. The main difference to the LWR cases is that the delayed neutron fraction uncertainty without the inclusion of the delayed nu-bar uncertainty is much larger than for LWR, and the increase caused by the delayed nu-bar uncertainty is much smaller. This means that the delayed neutron fraction uncertainty for the SFR assembly is most sensitive to reactions other than the delayed nu-bar. This is shown by systematic sensitivity analysis in the next subsection. Note however that the contribution of the delayed nu-bar uncertainty is still of the same order of magnitude as in the case of PWR and BWR, i.e. 2-2.5\%.

\subsection{Sensitivity analysis}

After having determined the uncertainties in the delayed neutron fractions due to all nuclear data uncertainties, and also the influence of the delayed nu-bar uncertainties by performing separate calculations without and with taking delayed nu-bar uncertainties into account, a systematic 
Table 1. Relevant contributors to the uncertainty of $\beta$ for the fuel assemblies at BOC end EOC.

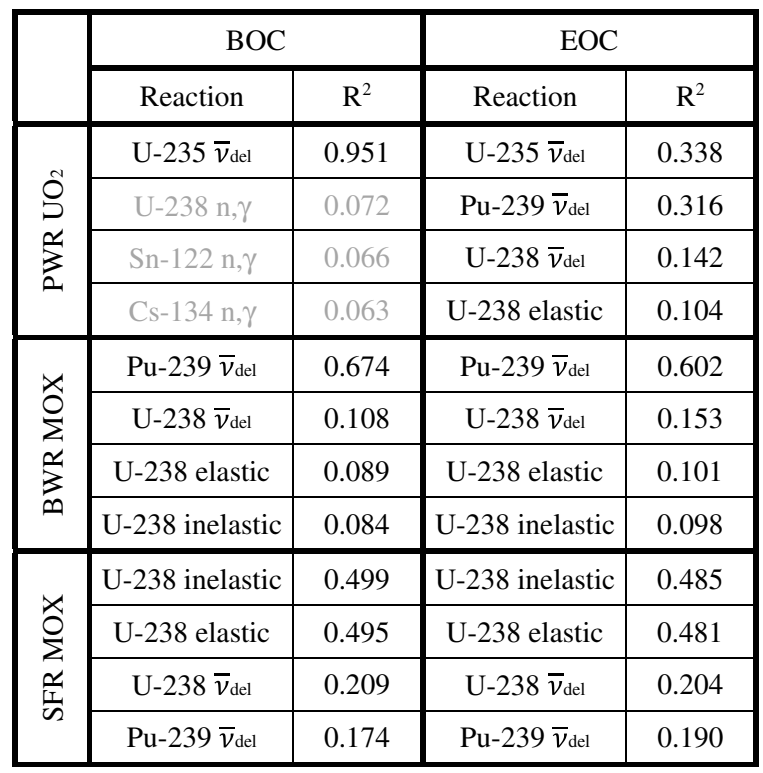

sensitivity analysis was performed as described in Sect. 2 to determine the main contributors to the result uncertainties.

In Table 1, the main contributors to the uncertainty of the delayed neutron fractions of the fuel assemblies are listed in terms of the squared multiple correlation coefficients $\mathrm{R}^{2}$. As already suggested by the results of the uncertainty analyses in Figs. 5 and 6, the main contributors for the LWR fuel assemblies are the uncertainties in the delayed nu-bar of U-235 and Pu-239 in the PWR $\mathrm{UO}_{2}$ and BWR MOX cases, respectively. For the PWR $\mathrm{UO}_{2}$ assembly at BOC, the uncertainty is largely dominated by the U-235 nu-bar uncertainty; although other contributors are listed in Table 1 (greyed out), they are only slightly above the $95 \%$ significance bound such that it is doubtful if they really contribute significantly to the uncertainty. For the SFR fuel assembly, the main contribution comes from U-238 scattering. In fact, the direct contributor is $\mathrm{U}-238$ inelastic scattering; the high $\mathrm{R}^{2}$ value of $\mathrm{U}-238$ elastic scattering is due to strong correlations between U-238 elastic and inelastic scattering cross sections. This was assessed by individually varying any of these reactions without taking all other reactions into consideration ("oneat-a-time" variation): as a result, the variation of the inelastic scattering practically accounts for the total $\beta$ uncertainty without delayed nu-bar (the blue curve in Fig. 7), whereas the variation of the elastic scattering gives almost no contribution to the $\beta$ uncertainty. Additional contributors are U-238 and Pu-239 delayed nu-bar with substantially lower $\mathrm{R}^{2}$; this explains why the inclusion of the delayed nu-bar uncertainties only yields a moderate increase of the delayed neutron fraction uncertainty.

Finally, the evolution of the importance indicators of the various delayed nu-bar uncertainties for the PWR $\mathrm{UO}_{2}$ fuel assembly is displayed as a function of operation time in Fig. 8. This clearly shows the dominance of the U-235 delayed nu-bar uncertainty for fresh fuel, decreasing with the depletion of U-235, and the increase of the Pu-239 delayed nu-bar contribution with the $\mathrm{Pu}-239$ build-up.

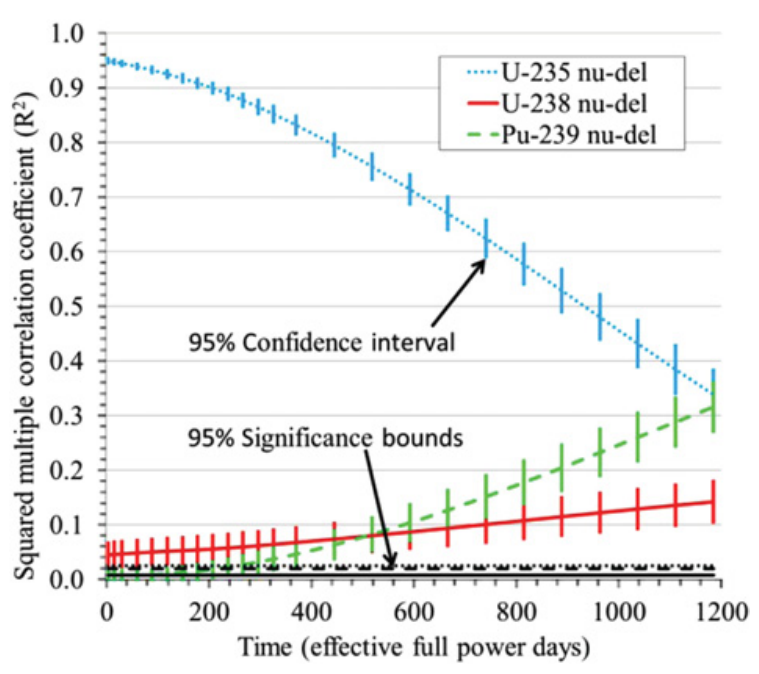

Figure 8. Importance indicators for the main contributors to the uncertainty of $\beta$ as a function of operation time for the $\mathrm{PWR} \mathrm{UO}_{2}$ fuel assembly.

\section{Conclusions and outlook}

Uncertainty and sensitivity analyses for the delayed neutron fractions for LWR and SFR fuel assemblies have been performed with XSUSA on the basis of the SCALE 6.1 covariance data supplemented by uncertainties in delayed nu-bar taken from JENDL-4.0. It was shown that the inclusion of these uncertainties leads to a substantial increase of the uncertainties in the delayed neutron fractions for LWR fuel, which can influence the uncertainties in subsequent core transient simulations. Note in addition that the contribution of the delayed nu-bar uncertainties is likely to be underestimated in some cases since no cross-correlations among different isotopes are included in JENDL-4.0 for these covariances. For SFR fuel, it turned out that the delayed neutron fraction uncertainty is dominated by the U-238 scattering uncertainty, and therefore the relative increase caused by the inclusion of delayed nu-bar uncertainties is smaller. In any case, to estimate the uncertainty of the dynamic behaviour of a reactor, uncertainties in the delayed nubar values should be taken into account when generating few-group cross sections and kinetic parameters in the corresponding lattice calculations. This is planned for later studies, in particular in the framework of the UAM-LWR benchmark.

The results in this paper are mainly based on SCALE 6.1 covariance data and JENDL-4 delayed nu-bar uncertainties. After performing these analyses, SCALE 6.2 has become available which contains ENDF/B-VII.1 based covariance data including individual values for prompt and delayed nu-bar. It is also planned to repeat the present studies on the basis of these data.

This work is supported by the German Federal Ministry for Economic Affairs and Energy.

\section{References}

[1] V. Mastrangelo, I. Kodeli, E. Sartori, "Proposal of a Benchmark for Stochastic and Deterministic Analyses of Nuclear Data Sensitivities and 
Uncertainties," Proc. Workshop on Uncertainty Analysis in Modelling, Coupled Multi-physics and Multi-scale LWR analysis (UAM-2006), Pisa, 28/29 April 2006, http://www.nea.fr/abs/html/ nea-1769.html

[2] K. Ivanov et al., "Benchmark for Uncertainty Analysis in Modelling (UAM) for Design, Operation and Safety Analysis of LWRs, Volume I," NEA/NSC/DOC(2012)10 (2016)

[3] I. Kodeli, M. Aufiero, W. Zwermann, "Comparison of Deterministic and Monte Carlo Codes SUSD3D, SERPENT and XSUSA for Beta-Effective Sensitivity Calculations," PHYSOR 2016, Sun Valley, ID, USA (2016)

[4] "International Handbook of Evaluated Criticality Safety Benchmark Experiments," Sept. 2015 Ed., available on DVD-ROM, NEA/NSC/DOC(95)03

[5] "International Handbook of Evaluated Reactor Physics Benchmark Experiments," March 2015 Ed., available on DVD-ROM, NEA/NSC/DOC(2006)1

[6] F. Bostelmann et al., "Sampling-Based Nuclear Data Uncertainty Analysis in Criticality and Depletion
Calculations," ANS MC2015, Nashville, TN, USA (2015)

[7] "Scale: A Comprehensive Modeling and Simulation Suite for Nuclear Safety Analysis and Design," ORNL/TM-2005/39, Version 6.1 (2011)

[8] K. Shibata et al., "JENDL-4.0: A New Library for Innovative Nuclear Energy Systems," Journal of the Korean Physical Society 59, 1046-1051 (2011)

[9] F. Bostelmann et al., "Uncertainty and Sensitivity Analysis in Criticality Calculations with Perturbation Theory and Sampling," ANS MC2015, Nashville, TN, USA (2015)

[10] N. Porter et al., "Benchmark for Uncertainty Analysis in Modelling (UAM) for Design, Operation and Safety Analysis of LWRs, Volume II," NEA/NSC/ DOC (2016)

[11] "Physics of Plutonium Fuels - BWR MOX Benchmark Specification and Results," OEDC/NEA 2003, ISBN: 92-64-19905-5

[12] D. Blanchet et al., "AEN - WPRS Sodium Fast Reactor Core Definitions," 2011, available from OECD/NEA Data Bank 